\title{
Immunohistochemical Pharmacokinetics of the Anti-diabetes Drug Alogliptin in Rat Kidney and Liver
}

\author{
Yutaro Yamamoto, Yuta Yamamoto, Tetsuya Saita, Daisuke Hira, Takahito Chijiwa and \\ Masashi Shin
}

Department of Applied Life Science, Faculty of Biotechnology and Life Science, Sojo University, 4-22-1 Ikeda, Nishi-ku, Kumamoto 860-0082, Japan

Received November 30, 2019; accepted April 30, 2020; published online May 29, 2020

\begin{abstract}
Alogliptin is one of a new class of therapeutic agents for type 2 diabetes called dipeptidyl peptidase-4 inhibitors. Here, we used immunohistochemistry to investigate the pharmacokinetics of alogliptin at the cell and tissue levels in the rat kidney and liver. One hour after alogliptin administration, the most noticeable immunoreactivity in the kidney was a moderate-to-strong staining in proximal tubule S3 segment epithelial cells. On the other hand, immunostaining was found only in the microvilli of S1 and S2 segment cells. Immunoreactivity was also observed in the glomerulus and distal tubules. Positive cells and almost negative cells coexisted in the collecting ducts. Twenty-four hours after administration, moderate immunostaining remained in the S3 segment but staining in other regions had almost disappeared. In the liver $1 \mathrm{hr}$ after administration, hepatocyte staining differed in the hepatic lobule, with zone III being stronger than zone I. Immunostaining had almost disappeared $24 \mathrm{hr}$ after administration. These findings suggest that alogliptin reabsorption at the kidney and uptake at the hepatocyte vary from region to region and that one or more types of transporter are involved in these processes. In addition, long-term alogliptin use may cause the drug to accumulate in S3 segment, leading to adverse events.
\end{abstract}

Key words: immunohistochemistry, pharmacokinetics, alogliptin, kidney, liver

\section{Introduction}

The number of diabetes patients around the world has steadily risen in recent decades [28]. The number of patients treated for diabetes in Japan reached a historical high of 3.3 million in 2017 [19], more than $90 \%$ of whom have type 2 diabetes mellitus. Alogliptin (AG) is one of a new class of therapeutic agents for type 2 diabetes called dipeptidyl peptidase-4 (DPP-4) inhibitors. When food enters the digestive tract, incretins, a type of gastrointestinal hormone, are secreted from the small intestine and promote insulin secretion from the pancreas. However,

Correspondence to: Masashi Shin, Department of Applied Life Science, Faculty of Biotechnology and Life Science, Sojo University, 4-22-1 Ikeda, Nishi-ku, Kumamoto 860-0082, Japan.

E-mail: mshin@life.sojo-u.ac.jp because incretins are rapidly degraded by DPP-4 in plasma, they cannot sufficiently stimulate a pancreas that has a diminished ability to secrete insulin. Alogliptin augments glucose-dependent insulin secretion by enhancing the action of incretins through DPP-4 inhibition [22]. Incretinbased therapies are considered safer than conventional drugs $[4,5,23]$. However, hepatic dysfunction with marked increases in, for example, blood AST (GOT), ALT (GPT), and AL-P, has been mentioned as a serious adverse event and, although the number of cases is low, kidney disorder, including chronic renal failure, has been reported $[6,26]$.

DPP-4 includes not only a soluble form circulating in the blood $[16,18]$, but also a membrane-bound form known as CD26, a lymphocyte surface antigen. This enzyme additionally shows high activity in vascular endothelial cells and in the small intestine, kidney, liver, and pancreas [10, $13,16]$. Thus, knowledge of the localization and accumula- 
tion of DPP-4 inhibitors in cells and tissues of animals would boost our understanding of the mechanisms underlying the action and/or adverse events of the drugs and of their appropriate usage. However, only a few reports on the cell and tissue localization of the DPP-4 inhibitors have been obtained, all involving autoradiography using radiolabeled drugs [11, 15, 21].

Pharmacokinetics comprises four processes: absorption, distribution, metabolism, and excretion. In a previous study, we reported the preparation of a specific monoclonal antibody (mAb) to AG and the localization of the drug in the rat small intestine, which is the main organ responsible for drug absorption, revealed by immunohistochemistry using the mAb [29]. In this study, we examined the localization of AG in the kidney and liver, which are the main organs for drug metabolism and excretion. First, we examined the localization at $1 \mathrm{hr}$ after administration to determine where the absorbed drug was localized in organs. Then, because AG is taken once-daily throughout life, its localization at $24 \mathrm{hr}$ after administration was studied to determine whether it accumulates due to long-term use.

\section{Materials and Methods}

\section{Antibody}

The anti-AG mAb was from the same batch used in our previous study (AAG-78) [29]. This $\mathrm{mAb}$ has high specificity for AG but does not react with other drugs such as sitagliptin, vancomycin, and amoxicillin [29].

\section{Animals}

In this study, we used normal adult male Wistar rats (Kyudo Exp. Animals, Kumamoto, Japan) weighing 200 250 g. This research was approved by the Animal Experiment Ethics Committee of Sojo University and was performed in accordance with standard ethical guidelines for the care and use of laboratory animals [24]. The animals were housed in temperature- and light-controlled rooms (21 $\pm 1^{\circ} \mathrm{C}$ and $12 \mathrm{hr}$ light:12 hr dark) and had free access to standard food and tap water. Six rats were orally administered a single dose of $5 \mathrm{mg} \mathrm{AG} / \mathrm{kg}$ of body weight. At $1 \mathrm{hr}$, $6 \mathrm{hr}$ and $24 \mathrm{hr}$, three rats each were anesthetized with sodium pentobarbital $(60 \mathrm{mg} / \mathrm{kg}$; Abbott Laboratories, North Chicago, IL, USA) and transcardially perfused with phosphate-buffered saline (PBS) containing 5,000 IU of heparin sodium (AY Pharmaceuticals Co., Ltd., Tokyo, Japan) at $50 \mathrm{ml} / \mathrm{min}$ for $2 \mathrm{~min}$ at room temperature and then with a freshly prepared solution of $2 \%$ glutaraldehyde in PBS for $6 \mathrm{~min}$. The kidney and liver were quickly excised and postfixed in the same fixative overnight at $4^{\circ} \mathrm{C}$.

\section{Immunohistochemistry}

Immunohistochemical staining was performed according to our previous methods with some modification [25, 29]. The postfixed specimens of the kidney and liver were embedded in paraffin according to standard protocols. The samples were cut into 5- $\mu$ m-thick sections and then deparaffinized and rehydrated. The sections were consecutively treated with (1) 6\% hydrogen peroxide (Nacalai Tesque, Kyoto, Japan) in PBS for $30 \mathrm{~min}$, (2) $2 \mathrm{~N} \mathrm{HCl}$ for $30 \mathrm{~min}$, (3) $0.03 \mathrm{mg} / \mathrm{ml}$ protease (Type XXIV: Bacterial; Sigma-Aldrich Co. Inc., St. Louis, MO, USA) in PBS for 0 or $60 \mathrm{~min}$ at $30^{\circ} \mathrm{C}$ [7], and (4) $5 \mathrm{mg} / \mathrm{ml} \mathrm{NaBH}_{4}$ (SigmaAldrich Co. Inc., St. Louis, MO, USA) in PBS for $10 \mathrm{~min}$ $[3,12]$. After each step, the specimens were washed three times with PBS. Next, the specimens were blocked with a protein solution containing 10\% normal goat serum, 1.0\% bovine serum albumin (BSA), and $0.1 \%$ saponin in Trisbuffered saline (TBS) for $1 \mathrm{hr}$ at room temperature and then directly incubated at $4^{\circ} \mathrm{C}$ overnight with AAG-78mAb, diluted 1:20 in TBS supplemented with $0.1 \%$ Triton X-100 (TBST). The sections were given three 5-min washes with TBST and then incubated with Simple Stain Rat MAX-PO (M) (Nichirei Bioscience Inc., Tokyo, Japan) for $2 \mathrm{hr}$ at room temperature. After TBS rinses, the site of the antigenantibody reaction was revealed by using the 3,3'diaminobenzidine tetrahydrochloride substrate (Dojindo Laboratories, Kumamoto, Japan) and $\mathrm{H}_{2} \mathrm{O}_{2}$ for 10 min.

Two types of negative control experiments - a conventional control experiment and an absorption control experiment-were performed. In the conventional control, the sections were exposed to normal mouse $\mathrm{IgG}$ diluted to 0.5 $\mu \mathrm{g} / \mathrm{ml}$ with TBST instead of the primary mAb. In the absorption control, the diluted primary $\mathrm{mAb}$ was preabsorbed with $30 \mu \mathrm{g} / \mathrm{ml}$ AG-GMBS-BSA, with a molar ratio more than 100-fold that of the antibody, before its reaction with the sections.

\section{Results}

\section{Localization of alogliptin in rat kidney}

Without protease treatment, the most noticeable staining was observed in the proximal tubule S3 segment of the kidney sections $1 \mathrm{hr}$ after AG administration (Fig. 1a, i). The microvilli of the epithelial cells were moderately stained and the nuclei strongly stained, whereas the cytoplasmic staining varied from moderately to strongly positive depending on the tubule cross-section (Fig. 1a). On the other hand, in the proximal tubule S1 and S2 segments, only weak staining was observed in the microvilli of the epithelial cells and neither the nucleus nor cytoplasm was stained (Fig. 1b). In addition, the distal tubules showed moderate staining (Fig. 1b). Both positive cells and almost negative cells were found in the collecting ducts (Fig. 1b). One hour of protease treatment barely changed the staining pattern and intensity of the proximal tubule S3 segments (Fig. 1c, j). However, the staining intensity of the microvilli of the epithelial cells of the proximal tubule S1 and S2 segments was markedly enhanced and the glomerulus was also positive (Fig. 1d, e). The staining intensity of distal tubules and collecting ducts was also slightly increased (Fig. 1d, e). In the kidney samples, which were treated with protease for 

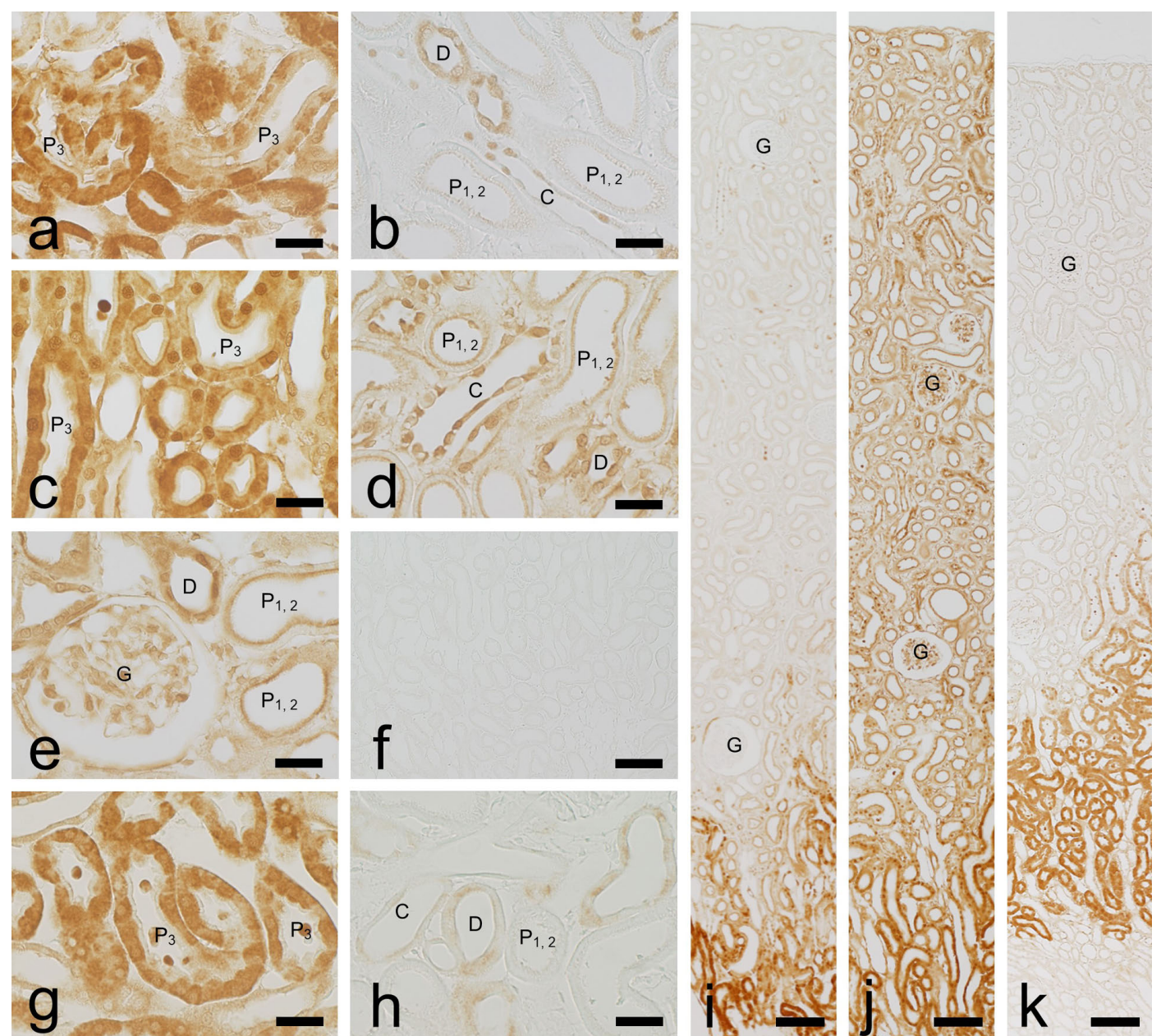

Fig. 1. Immunohistochemistry for AG in rat kidney. Tissue samples are from $1 \mathrm{hr}(\mathbf{a}-\mathbf{f}, \mathbf{i}, \mathbf{j})$ or $24 \mathrm{hr}(\mathbf{g}, \mathbf{h}, \mathbf{k})$ after drug administration. a, b, $\mathbf{g}-\mathbf{i}$ : Without protease digestion. $\mathbf{c}-\mathbf{f}, \mathbf{j}, \mathbf{k}$ : After digestion with protease for $1 \mathrm{hr} . \mathbf{a}, \mathbf{c}, \mathbf{g}$ : High-magnification images of the lower layer of the renal cortex. $\mathbf{b}, \mathbf{d}, \mathbf{e}, \mathbf{h}$ : High-magnification images of the upper layer of the renal cortex. f: Absorption control. G, glomerulus; $\mathrm{P}_{1,2}$, proximal tubule S1 or S2 segment; $\mathrm{P}_{3}$, proximal tubule S3 segment; D, distal tubule; C, collecting duct. Bars $=25 \mu \mathrm{m}(\mathbf{a}-\mathbf{e}, \mathbf{g}, \mathbf{h}) ; 100 \mu \mathrm{m}(\mathbf{f}, \mathbf{i}-\mathbf{k})$.

$1 \mathrm{hr}$, the staining intensity of proximal tubule S3 segments was hardly changed $6 \mathrm{hr}$ after administration whereas that of the other site was slightly reduced as compared with the sample at $1 \mathrm{hr}$ after administration (Supplementary Fig. S1).

When the protease treatment was not performed in the tissue $24 \mathrm{hr}$ after drug administration, the proximal tubule S3 segment staining was almost the same as that $1 \mathrm{hr}$ after administration (Fig. 1g). However, in the S1 and S2 segments, the epithelial cells, including their microvilli, were negative (Fig. 1h). The distal tubules showed weak staining, but the collecting duct was almost negative (Fig. 1h). One hour of protease treatment did not alter the staining and the glomerulus was negative (Fig. 1k).

\section{Localization of alogliptin in rat liver}

In the liver section $1 \mathrm{hr}$ after $\mathrm{AG}$ administration, when protease treatment was not performed, a very weak signal was observed in Kupffer cells and interlobular connective tissue, but hepatocytes were mostly unstained (data not shown). Liver sections treated with protease for $1 \mathrm{hr}$ showed weak-to-moderate staining throughout the samples (Fig. 2a). The staining intensity varied depending on the region of the hepatic lobule, and hepatocytes in zone III (area around the central vein) were slightly more intensely stained than those in zone I (peripheral area of the hepatic lobule) (Fig. 2a, b, c). In zone III hepatocytes, the nuclear staining was stronger than the cytoplasm (Fig. 2b). In addition, the endothelial cells of the interlobular artery, interlobular vein, and hepatic sinusoid and Kupffer cells were moderately positive and the interlobular bile duct epithelial cells were strongly positive (Fig. 2c). At $6 \mathrm{hr}$ after administration, the staining intensity at sites other than the interlobular bile duct was significantly reduced (Fig. 2d). At $24 \mathrm{hr}$ after administration, the staining in the liver almost disappeared (Fig. 2e).

\section{Negative control experiments}

The conventional immunohistochemical staining controls were all negative (data not shown). The absorption 

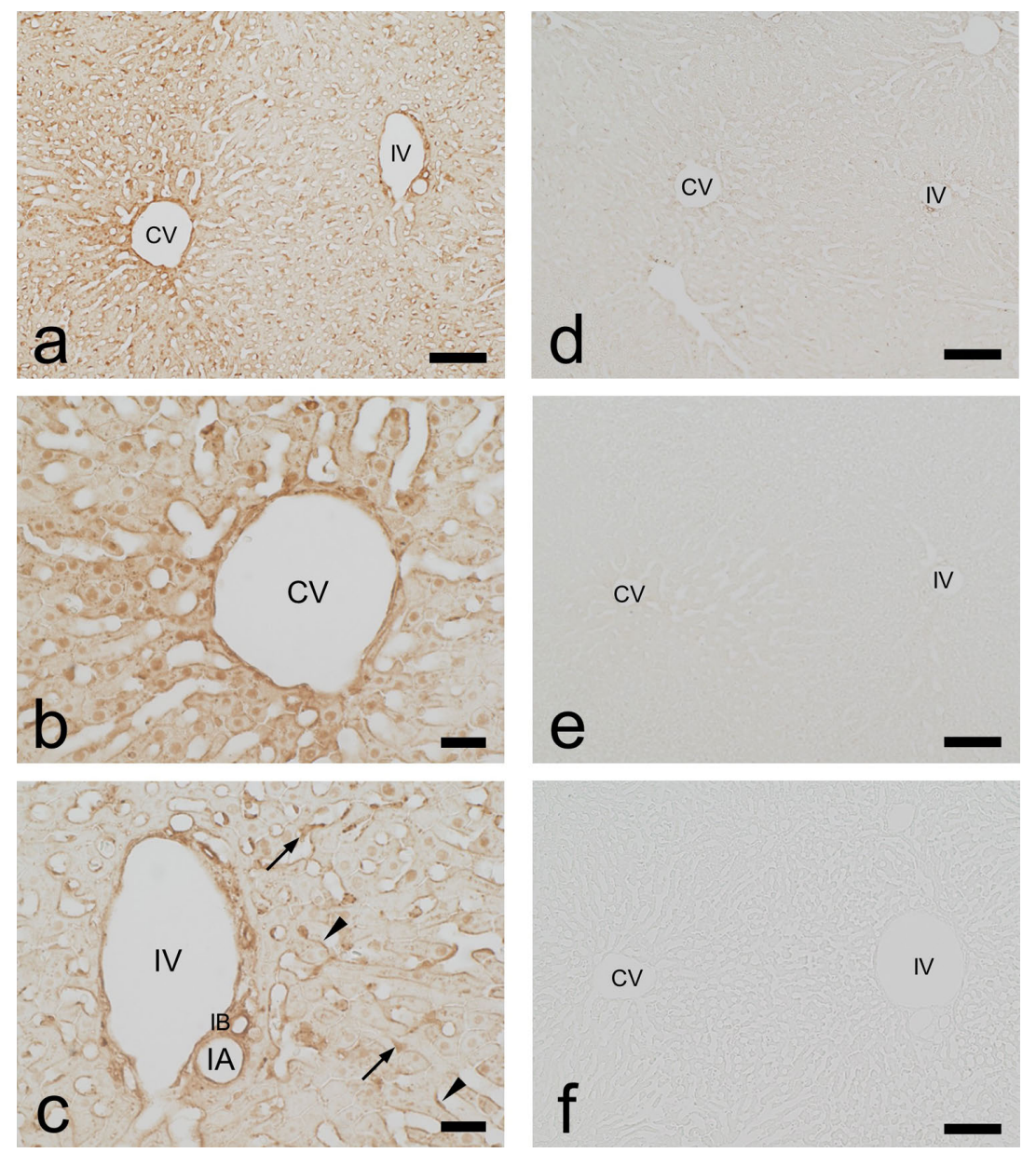

Fig. 2. Immunohistochemistry for $\mathrm{AG}$ in rat liver. Tissue samples are from $1 \mathrm{hr}(\mathbf{a}-\mathbf{c}, \mathbf{f}), 6 \mathrm{hr}(\mathbf{d})$ or $24 \mathrm{hr}(\mathbf{e})$ after drug administration. f: Absorption control. All samples were digested with protease for $1 \mathrm{hr}$. CV, central vein; IV, interlobular vein; IA, interlobular artery; IB, interlobular bile duct; arrows, Kupffer cells; arrow heads, endothelial cells. Bars $=100 \mu \mathrm{m}(\mathbf{a}, \mathbf{d}-\mathbf{f}) ; 25 \mu \mathrm{m}(\mathbf{b}, \mathbf{c})$.

controls showed that addition of AG-GMBS-BSA at a concentration of $30 \mu \mathrm{g} / \mathrm{ml}$ into the primary antibody solution abolished all staining (Figs. 1f, 2f).

\section{Discussion}

Drug excretion from the kidney is determined by the balance of glomerular filtration, tubular reabsorption, and tubular secretion. Therefore, immunostaining in tubular epithelial cells is believed to represent what remains between drug reabsorption from primary urine and drug secretion into it. Tubular reabsorption is generally considered to be based on passive transport and tubular secretion is considered to be due to active transport by transporters. However, PEPT1 and PEPT2 [1, 8, 14], which are transporters for di- and tripeptides, are involved in the reabsorption of $\beta$-lactam antibiotics. On the other hand, there are many transporters involved in tubular secretion, such as transporters that take up drugs from the basolateral side (e.g., organic anion transporters and organic cation transporters) $[14,20]$ and transporters that secrete drugs from the cell to the tubular lumen (e.g., P-glycoprotein and multidrug and toxin extrusion) $[14,20]$.

Immunohistochemistry for drugs requires glutaraldehyde fixation. Therefore, the intensity and localization of the signal obtained depends on the intensity of the protease digestion used to increase antibody permeability. Although increased protease digestion generally strengthens the signal intensity and reveals new positive sites, the signal observed in weakly digested or undigested sections is occasionally attenuated or absent [8]. In this study, even with protease treatment, only the microvilli of epithelial cells were stained in the renal proximal tubule S1 and S2 segments $1 \mathrm{hr}$ after AG administration. However, in the S3 segment cells, not only the microvilli, but also the cytoplasm and nuclei were stained without protease treatment. This indicates that a large amount of AG is localized at this site. As far as we know, transporters carrying AG as a substrate have not been reported, but our results suggest that not only passive transport, but also one or more types of transporter may be involved in the reabsorption of the drug and that the transporter may be highly expressed, particu- 
larly in S3 segment cells. A large amount of DPP-4 is expressed in the microvilli of proximal tubule epithelial cells [18]. Therefore, it is highly possible that the immunostaining in the microvilli shows not only the drug in transport, but also the drug bound to DPP-4.

In addition, the distal tubules showed moderate staining, and positive cells and almost negative cells coexisted in the collecting duct epithelium. To our knowledge, the only report on drug reabsorption or secretion in the distal tubules or collecting ducts is a report on the excretion of gentamicin from the distal tubule by Ueda et al. [27]. However, our results suggest that AG reabsorption is also performed in distal tubules and collecting ducts and that the uptake property is likely to vary from cell to cell. If the drug reabsorption at these regions were to occur only through passive transport, all epithelial cells would be similarly stained. Therefore, our findings suggest that $A G$ is reabsorbed from these regions via one or more types of transporter and that transporter expression varies from cell to cell. Because the collecting duct comprises two types of cells - principal cells and intercalated cells [17] - the difference in $A G$ accumulation may reflect the difference in cell types. In addition, the results of immunostaining for AG in the collecting duct were very similar to the results for vancomycin, but the swollen cells that occurred in vancomycin specimens were not observed. Because swollen cells are considered damaged cells, it is unlikely that AG damages the distal tubules and collecting ducts [9].

In the kidney $24 \mathrm{hr}$ after administration, there was almost no $A G$ in the region other than in the proximal tubule S3 segment, but a large amount of drug remained in the S3 segment. Therefore, it was suggested that long-term administration accumulated a large amount of the drug in the S3 segment and that the accumulated drug may be associated with adverse events in the kidney.

Alogliptin is partly metabolized by CYP2D6 and CYP3A4. However, the active metabolite M-I (Ndemethylated) accounts for less than $2 \%$ of the urinary AG concentration, and the inactive metabolite M-II (Nacetylated), which accounts for less than $6 \%$, has lost the amino group and thus cannot be fixed in tissues by glutaraldehyde $[2,6,23,26]$. Therefore, the immunostaining observed in the tissues is considered to indicate the localization of the unchanged drug. In the liver $1 \mathrm{hr}$ after AG administration, immunostaining was observed throughout the liver section, but the hepatocyte staining intensity was stronger in zone III than in zone I. In addition, the hepatocyte nuclei in zone III were strongly stained and granular positive reactions also occurred in the cells. These findings indicate that the amount of drug absorbed is higher for zone III hepatocytes than for zone I hepatocytes, although the blood drug concentration is higher in zone I sinusoid. It is highly advantageous for drug metabolism that more AG is taken up into zone III hepatocytes which strongly express drug-metabolizing enzymes. In addition, our results suggest that the uptake of AG into hepatocytes is not only based on passive transport, but also that some transporters are involved and that they are highly expressed in the hepatocytes in the center of the hepatic lobule. The immunostaining observed in vascular endothelial cells, bile capillaries, and interlobular bile duct epithelial cells seems to reflect AG bound to DPP-4 on the cell membranes of these cells. Alogliptin is mainly excreted from the kidney but may also be excreted in the bile to a minor extent. Because the immunostaining of hepatocytes almost disappears in the liver $24 \mathrm{hr}$ after administration, it is considered that $\mathrm{AG}$ in hepatocytes returns to the blood and does not accumulate in the cells, even after long-term use. Adverse events in the livers of AG users are likely not due to drug accumulation, but to other reasons.

In conclusion, our study clearly demonstrates the pharmacokinetics of AG at the cell and tissue levels in the rat kidney and liver. In the kidney, a large amount of the drug remains in the proximal tubule S3 segment, even $24 \mathrm{hr}$ after administration, suggesting that drug accumulation may be related to adverse events in the kidney. In the liver, zone III hepatocytes, which highly express drug-metabolizing enzymes, absorb more of the drug. However, the drug has almost disappeared from the hepatocytes $24 \mathrm{hr}$ after administration, which suggests that adverse events in the liver are due to reasons other than drug accumulation. Our results also suggest that one or more types of transporter are involved in drug uptake in these organs, in addition to passive transport. Immunohistochemistry using this $\mathrm{mAb}$ was useful for analyzing the localization of $A G$ in cells and tissues. In the future, we would like to study drug localization in various organs.

\section{Conflicts of Interest}

The authors declare no conflicts of interest.

\section{References}

1. Brandsch, M. (2009) Transport of drugs by proton-coupled transporters: pearls and pitfalls. Expert Opin. Drug Metab. Toxicol. 5; 887-905.

2. Christopher, R., Convingtone, P., Davenport, M., Fleck, P., Mekki, Q. A., Wann, E. R., et al. (2008) Pharmacokinetics, pharmacodynamics, and tolerability of single increasing does of the dipeptidyl peptidase- 4 inhibitor alogliptin in healthy male subjects. Clin. Ther. 30; 513-527.

3. Clancy, B. and Cauller, L. J. (1998) Reduction of background autofluorescence in brain sections following immersion in sodium borohydride. J. Neurosci. Methods 83; 97-102.

4. Drucker, D. J. and Nauck, N. A. (2006) The incretin system: glucagon-like peptide-1 receptor agonists and dipeptidyl peptidase-4 inhibitors in type 2 diabetes. Lancet 368; 1696-1705.

5. Egan, A. G., Blind, E., Dunder, K., de Graeff, P. A., Hummer, B. T., Bourcier, T., et al. (2014) Pancreatic safety of incretin-based drugs-FDA and EMA assessment. N. Engl. J. Med. 370; 794 797.

6. FDA (2020) FDA Adverse Event Reporting System (FAERS), Food and Drug Administration, Silver Spring, MD, USA. (always updating) 
7. Finley, J. C., Grossman, G. H., Dimeo, P. and Petrusz, P. (1978) Somatostatin-containing neurons in the rat brain: widespread distribution revealed by immunocytochemistry after pretreatment with pronase. Am. J. Anat. 153; 483-488.

8. Fujiwara, K., Shin, M., Miyazaki, T. and Maruta. Y. (2011) Immunocytochemistry for amoxicillin and its use for studying uptake of the drug in the intestine, liver, and kidney of rats. Antimicrob. Agents Chemother. 55; 62-71.

9. Fujiwara, K., Yoshizaki, Y., Shin, M., Miyazaki, T., Saita, T. and Nagata, S. (2012) Immunocytchemistry for vancomycin using a monoclonal antibody that reveals accumulation of the drug in tat kidney and liver. Antimicrob. Agents Chemother. 56; 5883-5891.

10. Fukasawa, K. M., Fukasawa, K., Sahara, N., Harada, M., Kondo, Y. and Nagatsu, I. (1981) Immunohistochemical localization of dipeptidyl aminopeptidase IV in rat kidney, liver, and salivary glands. J. Histochem. Cytochem. 29; 337-343.

11. Greischel, A., Binder, R. and Baierl, J. (2010) The dipeptidyl peptidase-4 inhibitor linagliptin exhibits time- and dosedependent localization in kidney, liver, and intestine after intravenous dosing: Results from high resolution autoradiography in rats. Drug Metab. Dispos. 38; 1443-1448.

12. Haraguchi, C. M. and Yokota, S. (2002) Immunofluorescence technique for 100-nm-thick semithin sections of Epon-embedded tissues. Histochem. Cell Biol. 117; 81-85.

13. Hartel, S., Gossrau, R., Hanski, C. and Reutter, W. (1988) Dipeptidyl peptidase (DPP) IV in rat organs. Comparison of immunohistochemistry and the activity histochemistry. Histochemistry 89; 151-161.

14. Inui, K., Masuda, S. and Saito, H. (2000) Cellular and molecular aspects of drug transport in the kidney. Kidney Int. 58; 944-958.

15. Klein, T., Fujii, M., Sandel, J., Shibazaki, Y., Wakamatsu, K., Mark, M., et al. (2014) Linagliptin alleviates hepatic steatosis and inflammation in a mouse model of non-alcoholic steatohepatitis. Med. Mol. Morphol. 47; 137-149.

16. Lambeir, A. M., Durinx, C., Scharpe, S. and De Meester, I. (2003) Dipeptidyl-peptidase IV from bench to bedside: an update on structural properties, functions, and clinical aspects of the enzyme DPP IV. Crit. Rev. Clin. Lab. Sci. 40; 209-294.

17. Madsen, K. M. and Tisher, C. C. (1986) Structural-functional relationship along the distal nephron. Am. J. Physiol. 250; F1-
F15.

18. Mentzel, S., Dijkman, H. B., Van Son, J. P., Koene, R. A. and Assmann, K. J. (1996) Organ distribution of aminopeptidase A and dipeptidyl peptidase IV in normal mice. J. Histochem. Cytochem. 1996; 445-461.

19. Ministry of Health, Labour and Welfare (2017) Summary of Patient Survey. (https://www.mhlw.go.jp/toukei/saikin/hw/kanja/ 17/index.html)

20. Motohashi, H., Nakano, Y., Masuda, S., Katsura, T., Kamba, T., Ogawa, O., et al. (2013) Precise comparison of protein localization among OCT, OAT, and MATE in human kidney. $J$. Pharm. Sci. 102; 3302-3308.

21. Nakamaru, Y., Akahoshi, F., Iiiima, H., Hisanaga, N. and Kume, T. (2016) Tissue distribution of teneligliptin in rats and comparisons with data reported for other dipeptidyl peptidase-4 inhibitors. Biopharm. Drug Dispos. 37; 142-155.

22. Saisho, Y. (2015) Alogliptin benzoate for management of type 2 diabetes. Vasc. Health Risk Manag. 11; 229-243.

23. Scheen, A. J. (2015) Safety of dipeptidyl peptidase-4 inhibitors for treating type 2 diabetes. Expert Opin. Drug Saf. 14; 505-524.

24. Science Council of Japan (2006) Guidelines for Proper Conduct of Animal Experiments.

25. Shin, M., Larsson, L.-I., Hougaard, D. M. and Fujiwara, K. (2009) Daunomycin accumulation and induction of programmed cell death in rat hair follicles. Cell Tissue Res. 337; 429-438.

26. Takeda Pharmaceutical Co., Ltd. (2019) Nesina (alogliptin), Interview Form.

27. Ueda, Y., Nakamura, N., Matsumoto, F., Tadokoro, H., Saito, A., Yamagata, M., et al. (1967) Studies on gentamicin absorption, excretion, mechanism of renal excretion and nephrotoxicity. Chemotherapy $15 ; 275-281$.

28. WHO (2016) Global Report on Diabetes. World Health Organization, Geneva.

29. Yamamoto, Y., Yamamoto, Y., Saita, T. and Shin, M. (2019) Immunohistochemistry for anti-diabetes drug, alogliptin using a newly prepared monoclonal antibody: Its precise localization in rat small intestine. Acta Histochem. Cytochem. 52; 27-34.

This is an open access article distributed under the Creative Commons Attribution License, which permits unrestricted use, distribution, and reproduction in any medium, provided the original work is properly cited. 